\title{
Microarray profiling defines circulating microRNAs associated with myelodysplastic syndromes
}

\author{
M. DOSTALOVA MERKEROVA*, A. HRUSTINCOVA, Z. KREJCIK, H. VOTAVOVA, E. RATAJOVA, J. CERMAK, M. BELICKOVA
}

Institute of Hematology and Blood Transfusion, Prague, Czech Republic

${ }^{*}$ Correspondence: michaela.merkerova@uhkt.cz

Received November 7, 2016 / Accepted January 31, 2017

\begin{abstract}
Circulating microRNAs (miRNAs) are non-coding RNAs secreted into body fluids, and aberrant levels of these miRNAs correlate with diseases of various origins, making them highly potential clinical biomarkers. We investigated the spectrum of circulating miRNAs in the plasma of myelodysplastic syndrome (MDS) patients to identify miRNAs showing discriminatory levels in the patients with different prognosis. Plasma samples were analyzed with microarrays to define miRNA profiles, and the deregulated miRNAs were further studied using droplet digital PCR. With regard to the prognosis, the levels of miR-27a-3p, miR-150-5p, miR-199a-5p, miR-223-3p and miR-451a were reduced in higher-risk MDS. Multivariate analysis indicated miR-451a level as an independent predictor of progression-free survival $(\mathrm{HR}=0.072, P=0.006)$ and revealed a significant association of miR-223-3p level with overall survival $(\mathrm{HR}=0.039, P=.032)$. Our data demonstrate that plasma levels of specific miRNAs are associated with MDS patient outcome and may add information beyond the currently used scoring systems.
\end{abstract}

Key words: circulating miRNA, myelodysplastic syndromes, plasma, progression, microarray

Myelodysplastic syndromes (MDS) are a heterogeneous group of malignant hematopoietic stem cell (HSC) disorders characterized by cytopenias, inefficient hematopoiesis, dysplasia in one or more myeloid cell lineages and an increased risk of development to acute myeloid leukemia (AML). Patient prognosis is predicted using the International Prognostic Scoring System (IPSS), which takes into account peripheral cytopenias, blast percentage, and cytogenetic aberrations. However, due to the high heterogeneity of MDS, development of molecular tools that can predict patient prognosis and outcomes is still necessary.

Recently, it was discovered that microRNA (miRNA) molecules are not only present in the cellular environment but also circulate in a wide variety of body fluids, including blood plasma. These extracellular molecules are not mere remnants of cellular degradation lacking specific functions, as the active transport of miRNAs between distant cells can play an important role in long distance cell-to-cell communication [1-3].

In cancers, monitoring of plasma miRNAs has the potential to allow earlier diagnosis and refine a prognosis or predict the response to therapy. To date, only a few studies have investigated circulating miRNAs in MDS [4-6]. Two papers $[4,5]$ focused on specific circulating miRNAs (miR-21, let$7 \mathrm{a}$, and $\mathrm{miR}-16$ ) that were preselected based on information about their deregulation in blood cells and their importance in similar diseases. Researchers monitored the plasma/ serum levels of these miRNAs and showed that their levels could serve as prognostic markers for $\operatorname{MDS}[4,5]$. A recently published study by Zuo et al. [6] is the only available study investigating the miRNA profile in MDS plasma thus far. This study profiled the expression of 800 human miRNAs in MDS plasma and identified a 7-miRNA signature (let-7a, miR-144, miR-16, miR-25, miR-451, miR-651, and miR-655) as an independent predictor of survival in MDS patients with normal karyotypes.

To extend the panel of molecular markers for the prediction of MDS patient outcomes, we studied the changes in the plasma miRNAome in MDS patients, irrespective of cytogenetic findings, using arrays covering more than 2,000 human miRNAs. We determined miRNAs whose levels discriminated lower-risk patients from those with higher-risk MDS and evaluated their prognostic significance in predicting patient outcomes. 


\section{Patients and methods}

Patients. The study included 40 peripheral blood plasma samples collected from patients with primary MDS with no known history of previous malignancy, chemotherapy or radiation therapy from the Institute of Hematology and Blood Transfusion. The patient's diagnosis was assessed according to the WHO classification criteria. None of the patients had received therapy for their disease or HSC transplantation (HSCT) prior to blood collection. Blood plasma samples from 20 age-matched healthy donors with no adverse medical histories were used as controls. Written informed consent was obtained from all tested subjects in accordance with the approval from the Institutional Review Board.

RNA extraction. Peripheral blood was collected in EDTA tubes, blood plasma was separated from peripheral blood by centrifugation, and the samples were stored at $-80^{\circ} \mathrm{C}$. The absence of hemolysis in the plasma samples was confirmed spectrophotometrically by measuring the free hemoglobin (oxyhemoglobin absorbance at $414 \mathrm{~nm}$ ) [7]. Plasma samples were further centrifuged at $12,000 \mathrm{~g}, 4^{\circ} \mathrm{C}$ for 10 minutes to dispose of cell debris. Total RNA was extracted from individual samples using a modified phenol-chloroform extraction method with Trizol LS reagent (Invitrogen, Basel, Switzerland) according to the procedure of Filková et al. (2014) [8] and was quantified with a Qubit 2.0 fluorometer (Thermo Fisher Scientific, Waltham, MA, USA).

Table 1. Patient characteristics.

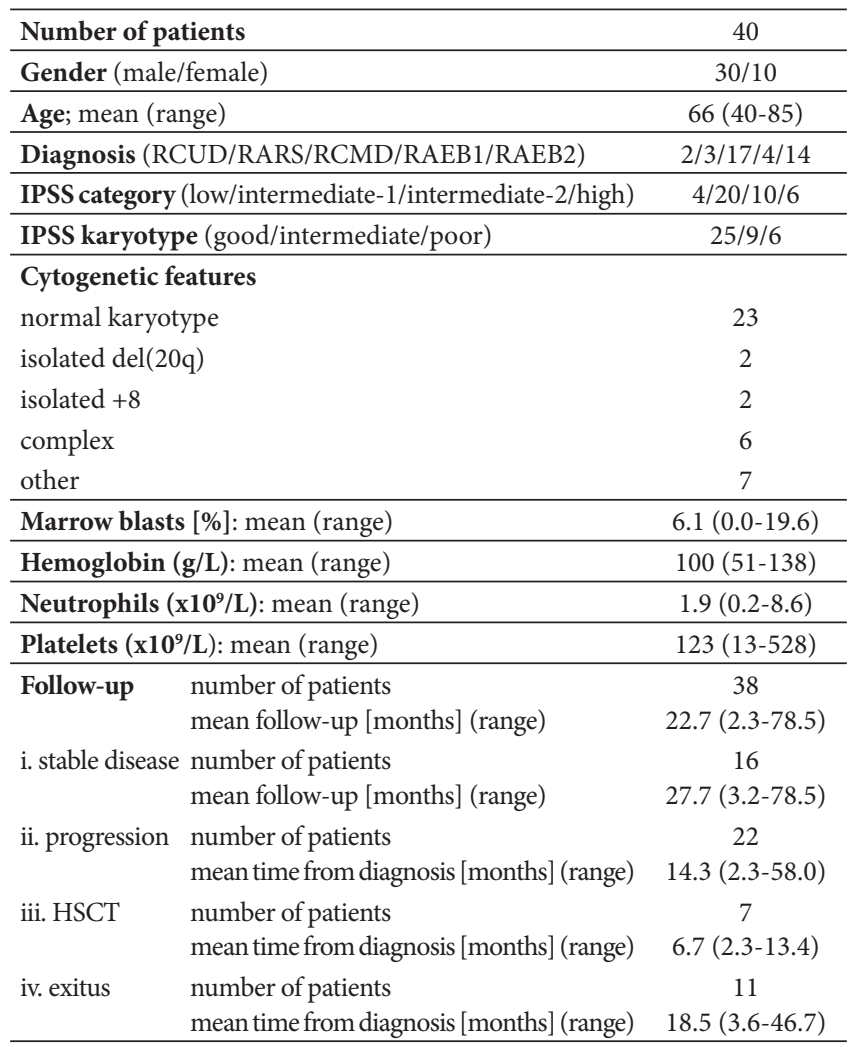

miRNA microarray profiling. Genome-wide miRNA profiles were determined using Agilent Human miRNA Microarrays (Sure Print G3 Unrestricted miRNA 8x60K, Release 19.0, Agilent Technologies, Santa Clara, CA, USA) in a discovery cohort (14 MDS patients and 7 healthy donors). The sample input was $350 \mathrm{ng}$ of total RNA. The samples were processed individually according to the manufacturer's protocol, and scanning was performed on an Agilent Microarray Scanner.

Quality control and background subtraction of raw microarray data were performed using Feature Extraction Software. Only those miRNAs whose signals were detected in more than 4 samples were included for further analysis. Quantile normalization and differential gene expression were performed in the $\mathrm{R}$ statistical environment (www.r-project.org) using standard statistical functions and the gtools package (|FC| and its modifications). Subsequent analysis of the microarray data was performed using $\mathrm{MeV}$ v4.8.1 software [9]. The Welch approximate t-test was applied to determine miRNAs differentially expressed between two groups of samples: (i) patients and controls and (ii) lower- and higher-risk patients. The complete raw and normalized data have been deposited in the NCBI Gene Expression Omnibus (GEO) database and are accessible through GEO Series accession number GSE76775.

Droplet digital PCR. The quantity of miR-16-5p, miR-27a-3p, miR-150-5p, miR-199a-5p, miR-223-3p, and miR-451a was verified in the validation cohort (all 40 patients) via droplet digital PCR (ddPCR) using QX200 ddPCR system (Bio-Rad, Hercules, CA, USA). Firstly, miRNAs were reverse-transcribed by TaqMan MicroRNA Reverse Transcription Kit (Life Technologies, Carlsbad, CA, USA) following manufacturer's instruction. Subsequently, PCR reaction was done with $2 \mathrm{X}$ ddPCR supermix for probes (Biorad) and 20X Taqman miRNA assay (Life Technologies). Droplets were prepared using QX200 Droplet Generator (Biorad) and PCR was performed under following conditions: $95^{\circ} \mathrm{C}$ for 10 minutes, then 40 cycles of $95^{\circ} \mathrm{C}$ for 15 seconds and $60{ }^{\circ} \mathrm{C}$ for 60 seconds, followed by enzyme deactivation at $98{ }^{\circ} \mathrm{C}$ for 10 minutes. Droplets with signal were counted by QX200 Droplet Reader (Biorad) and QuantaSoft software v. 1.6.6 (Biorad). A no template control and a negative control for each reverse transcription reaction were included in every assay.

Data analysis. Statistical analyses were performed using GraphPad Prism 4 (GraphPad Software, La Jolla, CA, USA) and SPSS (SPSS Inc., Chicago, IL) software. A Mann-Whitney test was used to compare levels of circulating miRNAs between different sample groups. The sensitivity and specificity of the optimum cut-off points were defined as the values that maximized the area under the ROC curve (AUC). The progression-free survival (PFS) and overall survival (OS) curves were generated by the Kaplan-Meier method, and the differences between groups were assessed by the log-rank test. Multivariate analysis was performed using the Cox proportional regression model. Pearson correlation analysis was 
performed to identify possible dependence between miRNA levels and blood count data.

\section{Results}

Characteristics of the study cohort. The study cohort included 40 plasma samples ( 30 males and 10 females) from primary MDS patients. The detailed clinical characteristics of all patients are summarized in Table 1. Patients' ages ranged between 40 and 85 years (mean 66 years), and their diagnoses according to the WHO criteria were as follows: refractory cytopenia with unilineage dysplasia (RCUD; $5 \% ; \mathrm{N}=2$ ), refractory anaemia with ringed sideroblasts (RARS; 7.5\%; $\mathrm{N}=3$ ), refractory cytopaenia with multilineage dysplasia (RCMD; $42.5 \%$; $\mathrm{N}=17$ ), refractory anaemia with excess blasts-1 (RAEB-1; $10 \% ; \mathrm{N}=4)$, and RAEB-2 $(35 \% ; \mathrm{N}=14)$. Karyotyping revealed normal karyotypes in 23 patients (57.5\%). Various chromosomal abnormalities were found in 17 patients (42.5\%; e.g., isolated del(20q) -2 patients, trisomy $8-2$ patients, complex karyotype -6 patients). According to the IPSS scoring system, the cytogenetic risk was good in $62.5 \%(\mathrm{~N}=25)$, intermediate in $22.5 \%(\mathrm{~N}=9)$, and poor in $15 \%(\mathrm{~N}=6)$ cases, and patients were stratified into low $(10 \%, \mathrm{~N}=4)$, intermediate- $1(50 \%$, $\mathrm{N}=20)$, intermediate-2 (25\%, $\mathrm{N}=10)$, and high (15\%, $\mathrm{N}=$ 6) IPSS categories. For expression analyses, the patients were further divided into a lower-risk MDS group (low /intermediate-1 IPSS categories, $\mathrm{N}=24$ ) and a higher-risk MDS group (intermediate-2/high IPSS categories, $\mathrm{N}=16$ ).

Table 2. The most abundant miRNAs in plasma of MDS patients and healthy donors

\begin{tabular}{rlclc}
\hline & MDS patients & $\begin{array}{c}\text { Signal intensity } \\
\text { (mean) }\end{array}$ & Healthy donors & $\begin{array}{c}\text { Signal intensity } \\
\text { (mean) }\end{array}$ \\
\hline 1 & miR-6089 & 25625 & miR-4454 & 23779 \\
2 & miR-4454 & 11048 & miR-6089 & 14383 \\
3 & miR-6125 & 9097 & miR-451a & 6069 \\
4 & miR-4516 & 4895 & miR-6090 & 5327 \\
5 & miR-6090 & 4797 & miR-223-3p & 4575 \\
6 & miR-2861 & 4414 & miR-3960 & 3652 \\
7 & miR-3960 & 3892 & miR-6125 & 3605 \\
8 & miR-638 & 3724 & let-7b-5p & 3151 \\
9 & miR-4459 & 2711 & miR-4516 & 2510 \\
10 & miR-3665 & 1805 & miR-21-5p & 2494 \\
11 & miR-1234-5p & 1775 & miR-2861 & 1876 \\
12 & miR-6068 & 1774 & miR-5100 & 1788 \\
13 & miR-4281 & 1698 & miR-16-5p & 1678 \\
14 & miR-1915-3p & 1622 & let-7a-5p & 1641 \\
15 & miR-630 & 1579 & miR-4459 & 1407 \\
16 & miR-371b-5p & 1533 & miR-4281 & 1271 \\
17 & miR-451a & 1351 & miR-6087 & 1205 \\
18 & miR-6087 & 1309 & miR-638 & 1162 \\
19 & miR-5703 & 1267 & let-7f-5p & 1129 \\
20 & miR-5100 & 1207 & miR-92a-3p & 1092 \\
\hline
\end{tabular}

Expression profiling of circulating miRNAs in MDS plasma. Genome-wide miRNA profiling was performed in the discovery cohort, and among the 2,006 miRNAs present on the array, 207 and 201 miRNAs were detectable in the MDS and control samples, respectively. The miRNAs that showed the highest expression levels in patients and healthy donors are listed in Table 2. Comparative analysis of the patient and control groups of samples identified 48 miRNAs showing significantly $(P<.01)$ altered levels in the plasma of MDS patients (irrespective of their clinical parameters) compared to their expression levels in control plasma (Figure 1 and Suppl

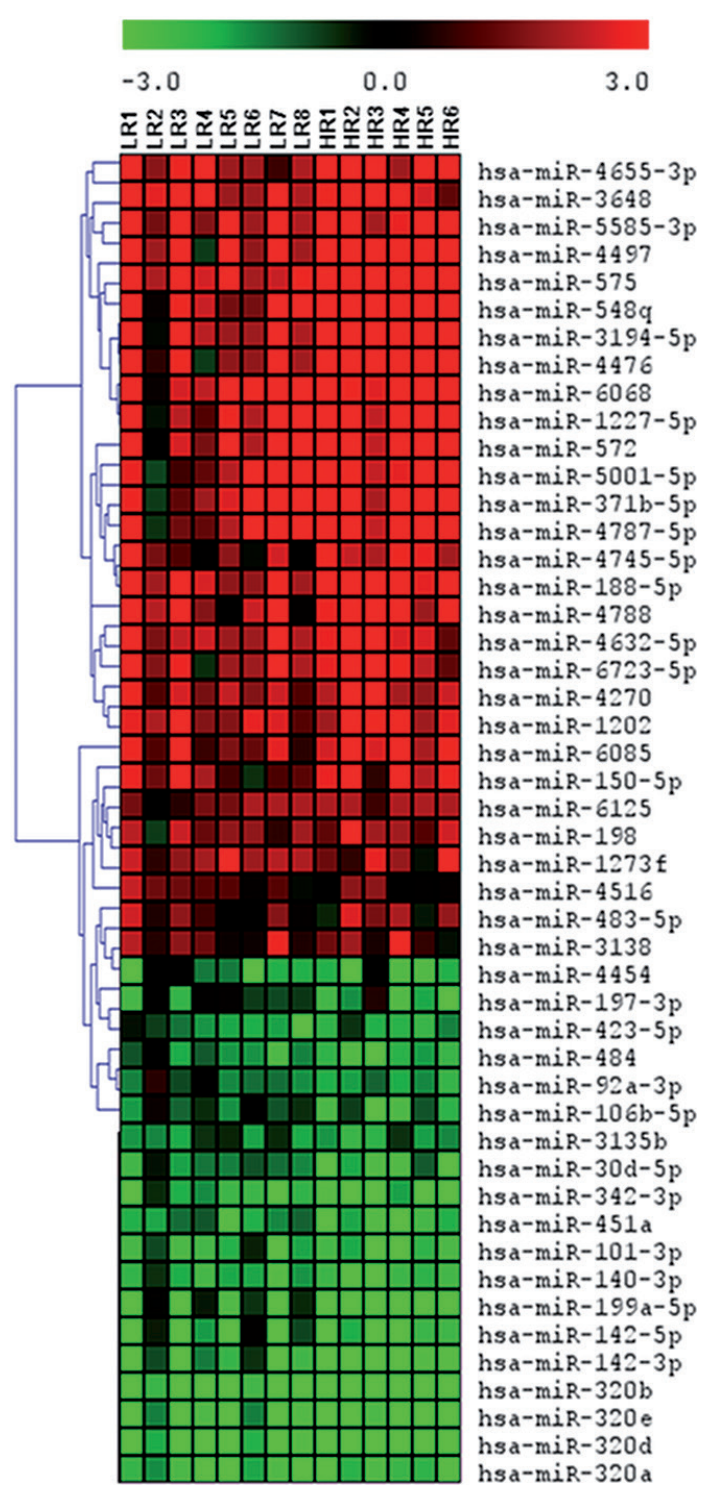

Figure 1. MiRNAs deregulated in MDS plasma compared to controls. Heatmap shows only the differentially expressed plasma miRNAs $(P<.01)$ in all MDS patients compared to controls. Color gradient intensity scale indicates fold change (binary logarithm) of signal intensities compared with the mean signal intensity of controls. red - increase, green - decrease, LR - lower-risk MDS patient, HR - higher-risk MDS patient. 


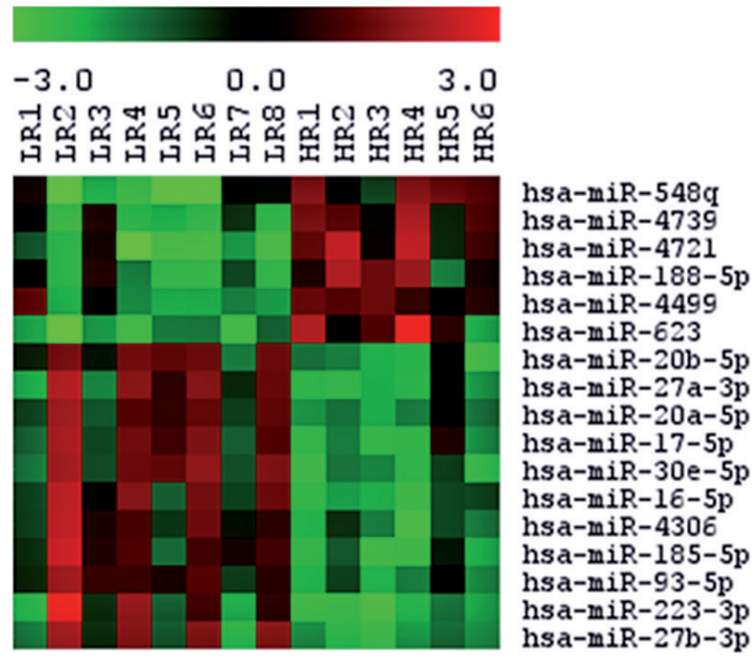

Figure 2. MiRNAs altered between lower-risk and higher-risk MDS. Heatmap shows differentially expressed plasma miRNAs $(P<.01)$ between lower-risk and higher-risk MDS patients. Color gradient intensity scale indicates fold change (binary logarithm) of signal intensities compared with the mean signal intensity for all patient samples. red - increase, green - decrease, LR - lower-risk MDS patient, HR - higher-risk MDS patient.

Table 1). Among those miRNAs, 19 were downregulated (e.g., miR-451a, miR-92a-3p, miR-320a/b/d/e, and miR-142-3p/5p), and 29 were upregulated (e.g., miR-150-5p, miR188-5p, and miR-371b-5p) in the patients.

The patients were further divided into a lower-risk category $(\mathrm{N}=8)$ and a higher-risk category $(\mathrm{N}=6)$. Statistical testing was performed to compare expression profiles of plasma miRNAs between these two groups, and 17 miRNAs were identified as differently expressed (e.g., miR-16-5p, miR-17$5 \mathrm{p}, \mathrm{miR}-27 \mathrm{a}-3 \mathrm{p} / \mathrm{b}-3 \mathrm{p}, \mathrm{miR}-223-3 \mathrm{p}$ were downregulated and miR-188-5p, miR-623 were upregulated in higher-risk MDS compared to lower-risk MDS) (Figure 2 and Suppl Table 2).

Quantification of individual circulating miRNAs by droplet digital PCR. Based on the microarray results, we selected six hematopoiesis- and/or oncology-related miRNAs (miR-16-5p, miR-27a-3p, miR-150-5p, miR-199a-5p, miR-223-3p, and miR-451a) that showed deregulated levels in MDS plasma and quantified their levels via ddPCR in the validation cohort. In concordance with the microarray data, plasma miR-150-5p was increased and miR-16-5p, miR-27a$3 \mathrm{p}$, miR-199a-5p, and miR-451a were reduced in MDS patients compared to healthy controls. Comparison of miRNA levels between higher-risk and lower-risk groups revealed significantly $(P<.05)$ lower levels of miR-27a-3p, miR-199a-5p, and miR-223-3p in higher-risk disease (Figure 3).

Correlation of miRNA levels with clinical variables. A series of univariate analyses was performed for various clinical variables and for each of the selected miRNAs to evaluate whether their plasma levels were potential predictors of patient outcomes. The mean patient follow-up was 22.7 months, and within this time period, 16 patients exhibited stable disease; 22 patients progressed; and 11 patients died. The univariate analysis indicated significantly different PFS $(P<.05)$ associated with the following parameters: IPSS karyotype, IPSS score, amount of cytopenias, WHO-based diagnosis, and the levels of five miRNAs (miR-27a-3p, miR-
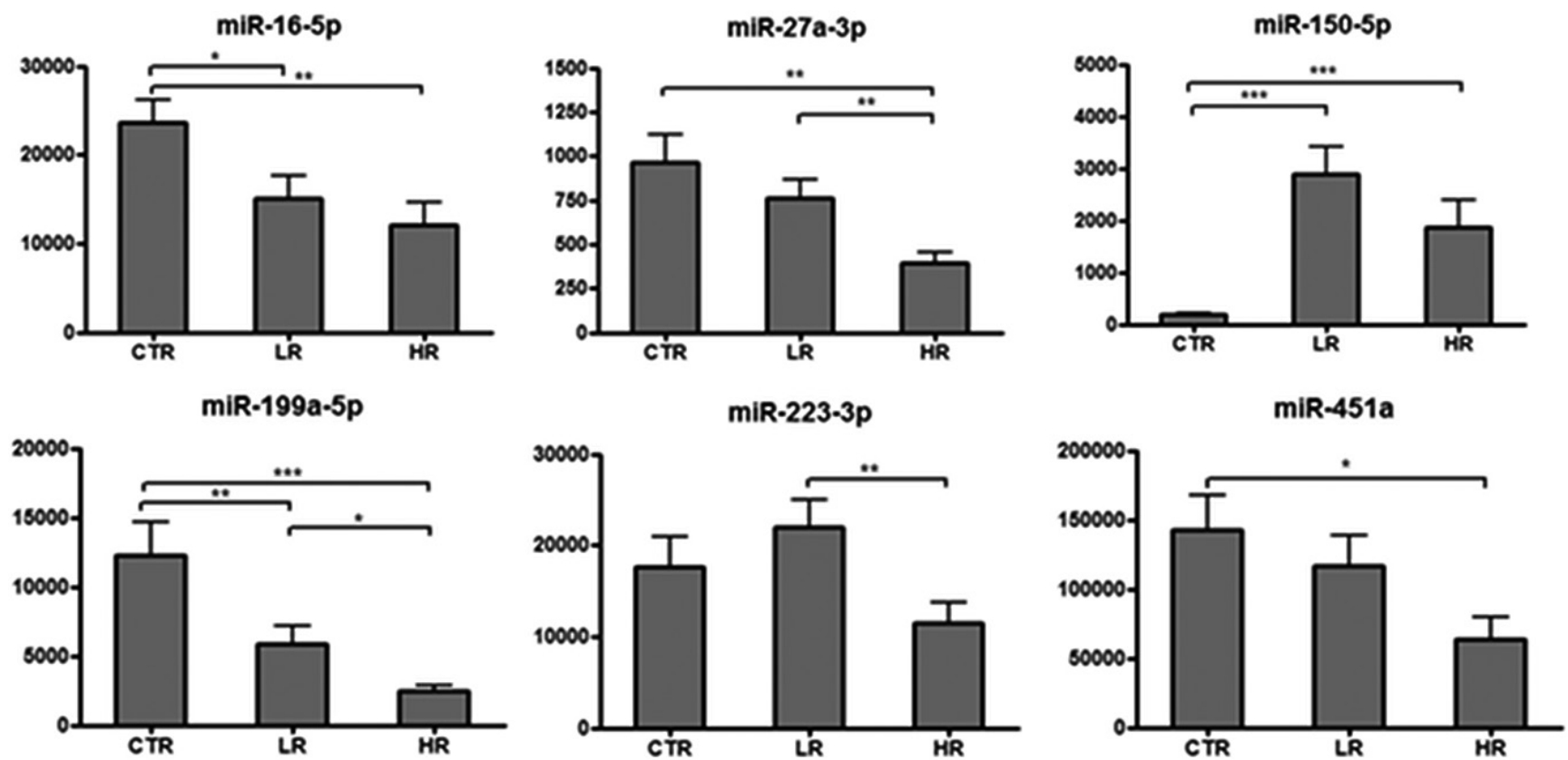

Figure 3. Level of plasma miRNAs. Absolute amounts of plasma miR-16-5p, miR-27a-3p, miR-150-5p, miR-199a-5p, miR-223-3p, and miR-451a were quantified via ddPCR. CTR - control, LR - lower-risk, HR - higher-risk MDS, ${ }^{\star} P<.05,{ }^{\star *} P<.01,{ }^{\star * \star} P<.001$. 
150-5p, miR-199a-5p, miR-223-3p, and miR-451a). The univariate analysis for OS showed distinct stratification of MDS patients based on miR-27a-3p and miR-223-3p plasma levels. The cut-off values for each miRNA assessed based on ROC curve analysis, mean PFS and OS values, 95\% confidence intervals (CIs) and $P$ values are listed for each of the tested variables in Table 3. Multivariate Cox analyses confirmed that the miR-451a plasma level (cut off $100 \times 10^{4}$ copies/ $\mu$ of plasma) and amount of cytopenias were associated with PFS and that the plasma level of miR-223-3p (cut off $17 \times 10^{4}$ copies/ $\mu \mathrm{l}$ of plasma) was associated with OS (Table 4). Kaplan-Meier curves showing that patients with low miR-451a expression exhibited significantly decreased PFS (25.7 months, 95\% CI 10.4 to 41.1 ) compared with patients with high expression
(41.5 months, $95 \%$ CI 29.1 to 54.0 ) and that patients with low miR-223-3p expression presented significantly decreased OS (27.7 months, 95\% CI 13.4 to 41.9 months) compared with patients with high expression (70.6 months, 95\% CI 57.1 to 84.1 months) are included in Figure 4.

To examine the possible origin of circulating miRNAs in different blood cell populations, we performed a correlation analysis for the six selected miRNAs with blood count data (numbers of erythrocytes, platelets, neutrophils, monocytes, leukocytes, and lymphocytes; hemoglobin concentration; and percentage of blasts in bone marrow). However, we did not detect significant correlations between the levels of any of the selected miRNAs and blood counts of the tested lineages.

Table 3. Univariate analysis for progression-free survival and overall survival.

\begin{tabular}{|c|c|c|c|c|c|c|c|}
\hline \multirow[t]{2}{*}{ Variable } & & \multicolumn{3}{|c|}{ Progression-free survival } & \multicolumn{3}{|c|}{ Overall survival } \\
\hline & & Mean est. (mo) & $95 \% \mathrm{CI}$ & $P$ value & $\begin{array}{c}\text { Mean } \\
\text { est. (mo) }\end{array}$ & $95 \%$ CI & $P$ value \\
\hline \multirow[t]{2}{*}{ Age } & $<65$ years & 26.0 & $9.5-42.4$ & 0.155 & 52.1 & $29.9-74.3$ & 0.739 \\
\hline & $\geq 65$ years & 35.9 & $23.3-46.6$ & & 42.6 & $27.6-57.6$ & \\
\hline \multirow[t]{2}{*}{ Gender } & male & 36.8 & $23.3-50.4$ & 0.375 & 46.3 & $33.0-65.6$ & 0.507 \\
\hline & female & 23.8 & $8.6-39.1$ & & 49.3 & $29.4-63.1$ & \\
\hline \multirow[t]{3}{*}{ Diagnosis } & RCUD/ RARS & 41.7 & $19.5-63.9$ & 0.038 & 41.7 & $19.5-63.9$ & 0.958 \\
\hline & RCMD & 41.2 & $24.6-57.8$ & & 50.6 & $32.4-68.9$ & \\
\hline & RAEB-1/ RAEB-2 & 13.2 & $7.5-18.9$ & & 26.8 & $20.1-33.5$ & \\
\hline \multirow[t]{2}{*}{ IPSS category } & lower-risk & 42.2 & $27.9-56.4$ & 0.013 & 50.8 & $35.2-66.5$ & 0.709 \\
\hline & higher-risk & 12.7 & $6.9-18.6$ & & 26.3 & $19.2-33.5$ & \\
\hline \multirow[t]{3}{*}{ IPSS karyotype } & good & 42.5 & $27.9-57.2$ & 0.005 & 53.6 & $36.8-70.5$ & 0.082 \\
\hline & intermediate & 24.7 & $10.6-38.9$ & & 42.0 & $30.7-53.3$ & \\
\hline & poor & 6.4 & $3.3-9.6$ & & 10.2 & $6.4-13.9$ & \\
\hline \multirow[t]{2}{*}{ Blasts } & $<5 \%$ & 38.5 & $24.0-53.0$ & 0.191 & 45.3 & $28.9-61.7$ & 0.314 \\
\hline & $\geq 5 \%$ & 22.0 & $8.8-35.1$ & & 47.6 & $28.4-66.8$ & \\
\hline \multirow[t]{2}{*}{ Hemoglobin } & $<100 \mathrm{~g} / \mathrm{l}$ & 26.2 & $12.9-39.5$ & 0.082 & 48.5 & $29.5-67.5$ & 0.699 \\
\hline & $\geq 100 \mathrm{~g} / 1$ & 37.0 & $22.7-51.2$ & & 42.3 & $27.7-57.0$ & \\
\hline \multirow[t]{2}{*}{ Neutrophils } & $<1.5 \times 10^{9} / 1$ & 29.4 & $15.1-43.7$ & 0.467 & 40.7 & $24.1-57.3$ & 0.183 \\
\hline & $\geq 1.5 \times 10^{9} / 1$ & 34.5 & $20.7-48.3$ & & 59.7 & $46.9-72.4$ & \\
\hline \multirow[t]{2}{*}{ Platelets } & $<150 \times 10^{9} / 1$ & 27.3 & $15.0-39.5$ & 0.126 & 43.8 & $26.7-60.7$ & 0.179 \\
\hline & $\geq 150 \times 10^{9} / 1$ & 38.8 & $23.2-54.4$ & & 49.6 & $32.4-66.8$ & \\
\hline \multirow[t]{2}{*}{ Amount of cytopenia } & $0-1$ lineage & 46.8 & $31.0-62.5$ & 0.024 & 59.0 & $49.2-68.7$ & 0.084 \\
\hline & $2-3$ lineages & 24.9 & $13.7-36.1$ & & 40.0 & $23.8-56.2$ & \\
\hline \multirow[t]{2}{*}{ miR-16-5p plasma level } & $<12 \times 10^{4}$ copies $/ \mu \mathrm{l}$ of plasma & 25.6 & $11.4-39.8$ & 0.338 & 49.8 & $29.1-70.4$ & 0.769 \\
\hline & $\geq 12 \times 10^{4}$ copies $/ \mu \mathrm{l}$ of plasma & 33.6 & $20.3-47.0$ & & 41.8 & $25.7-58.0$ & \\
\hline \multirow[t]{2}{*}{ miR-27a-3p plasma level } & $<2.7 \times 10^{3}$ copies $/ \mu$ of plasma & 11.2 & $6.2-16.2$ & 0.005 & 18.0 & $11.8-24.1$ & 0.001 \\
\hline & $\geq 2.7 \times 10^{3} \mathrm{copies} / \mu \mathrm{l}$ of plasma & 41.9 & $28.0-55.8$ & & 60.7 & $45.7-75.6$ & \\
\hline \multirow[t]{2}{*}{ miR-150-5p plasma level } & $<25 \times 10^{3}$ copies $/ \mu \mathrm{l}$ of plasma & 26.9 & $14.0-40.0$ & 0.038 & 44.9 & $27.6-62.2$ & 0.089 \\
\hline & $\geq 25 \times 10^{3}$ copies $/ \mu 1$ of plasma & 50.1 & $36.0-64.1$ & & 60.0 & $43.4-76.4$ & \\
\hline \multirow[t]{2}{*}{ miR-199a-5p plasma level } & $<2.6 \times 10^{3}$ copies $/ \mu$ of plasma & 21.9 & $8.9-34.9$ & 0.048 & 37.9 & $19.4-56.5$ & 0.100 \\
\hline & $\geq 2.6 \times 10^{3}$ copies $/ \mu$ of plasma & 37.4 & $24.3-50.5$ & & 47.6 & $30.9-64.4$ & \\
\hline \multirow[t]{2}{*}{ miR-223-3p plasma level } & $<17 \times 10^{4}$ copies $/ \mu$ of plasma & 18.3 & $9.0-27.7$ & 0.008 & 27.7 & $13.4-41.9$ & 0.001 \\
\hline & $\geq 17 \times 10^{4}$ copies $/ \mu \mathrm{l}$ of plasma & 49.6 & $32.1-67.2$ & & 70.6 & $57.1-84.1$ & \\
\hline \multirow[t]{2}{*}{ miR-451a plasma level } & $<100 \times 10^{4}$ copies $/ \mu$ of plasma & 25.7 & $10.4-41.1$ & 0.029 & 56.3 & $38.6-74.1$ & 0.709 \\
\hline & $\geq 100 \times 10^{4}$ copies $/ \mu 1$ of plasma & 41.5 & $29.1-54.0$ & & 47.6 & $33.3-61.9$ & \\
\hline
\end{tabular}



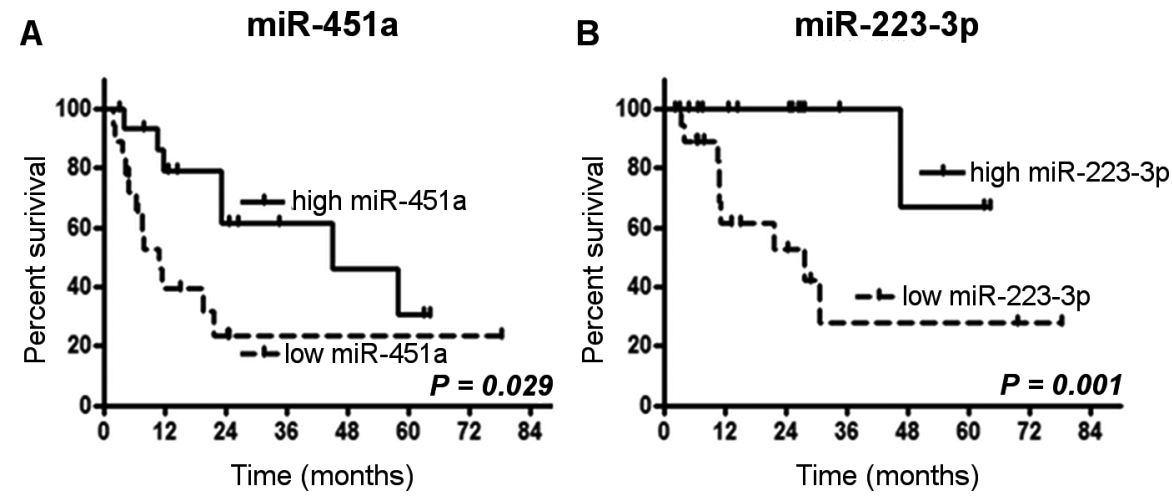

Figure 4. Patient survival according to miRNA levels. Kaplan-Meier curves for progression-free survival based on miR-451a plasma level (A) and overall survival according to miR-223-3p plasma level (B).

Expression of the selected miRNAs in CD34+ cells. Previously, we assessed miRNA signatures in CD34+ MDS cells through microarray profiling [10]. Here, we employed these data to evaluate the cellular levels of the plasma-related miRNAs (miR-16-5p, miR-27a-3p, miR-150-5p, miR-199a-5p, miR-223-3p, and miR-451a) with regard to patient prognosis. We observed some expression changes between controls, higher-risk, and lower-risk patient subgroups (Suppl Figure 1); however, we did not found any apparent relations of intra- and extracellular miRNA levels.

\section{Discussion}

Due to stability and accessibility, circulating miRNAs represent promising non-invasive cancer biomarkers. Numerous studies have demonstrated their ability to accurately classify discrete tumor types and disease stages. In this study, we defined specific miRNA profiles in MDS plasma and demonstrated that extracellular miRNAs may also be used for effective stratification of MDS patients with different prognoses.

To address the association of plasma miRNAs with patient prognosis, we assayed the levels of MDS-related miRNAs
(miR-16-5p, miR-27a-3p, miR-150-5p, miR-199a-5p, miR223-3p, and miR-451a) selected based on our microarray results. Comparison of the miRNA plasma levels between higher-risk and lower-risk groups of patients suggested an association of the decreased levels with adverse prognosis. With respect to the premise that circulating miRNAs serve as active extracellular cell-to-cell communicators, reduction of multiple hematopoiesis-related miRNAs in plasma should presumably contribute to a more progressive failure of the entire hematopoietic process.

Using ROC curve analysis, we defined optimal cut-off points for each of these miRNAs and evaluated their prognostic value for PFS and OS. We showed that the low level of miR-451a is an independent predictor of reduced PFS, and the low level of miR-223-3p associates significantly with reduced OS. These results indicate that the levels of specific plasma miRNAs may predict MDS patient outcomes independent of other criteria.

The physiological roles of circulating miRNAs and the reasons of their deregulation in MDS are unclear. However, it is known that cellular miR-451a and miR-223 are strong regulators of the hematopoiesis and target differentiation of blood

Table 4. Multivariate Cox analysis for progression-free survival and overall survival.

\begin{tabular}{|c|c|c|c|c|c|c|}
\hline \multirow[b]{2}{*}{ Variable } & \multicolumn{3}{|c|}{ Progression-free survival } & \multicolumn{3}{|c|}{ Overall survival } \\
\hline & $\mathrm{HR}$ & $95 \% \mathrm{CI}$ & $\mathrm{P}$ value & HR & $95 \% \mathrm{CI}$ & P value \\
\hline Diagnosis & 0.512 & $0.040-6.488$ & 0.300 & 0.923 & $0.148-5.744$ & 0.932 \\
\hline IPSS category & 6.398 & $0.835-49.032$ & 0.074 & 1.089 & $0.089-13.369$ & 0.947 \\
\hline IPSS karyotype & 1.207 & $0.358-4.064$ & 0.762 & 0.151 & $0.014-1.693$ & 0.125 \\
\hline Amount of cytopenia & 12.647 & $1.578-101.358$ & 0.017 & 2.128 & $0.172-26.287$ & 0.556 \\
\hline miR-27a-3p & 0.177 & $0.026-1.201$ & 0.076 & 0.682 & $0.103-4.511$ & 0.691 \\
\hline miR-150-5p & 2.983 & $0.376-23.685$ & 0.301 & n.a. & n.a. & n.a. \\
\hline miR-199a-5p & 4.133 & $0.527-32.404$ & 0.177 & n.a. & n.a. & n.a. \\
\hline miR-223-3p & 0.739 & $0.155-3.513$ & 0.704 & 0.039 & $0.002-0.856$ & 0.032 \\
\hline miR-451a & 0.072 & $0.011-0.467$ & 0.006 & n.a. & n.a. & n.a. \\
\hline
\end{tabular}

HR - hazard ratio, CI - confidence interval, n.a. - not analyzed 
cells as well as their proliferation and apoptosis in many ways. miR-451 is a positive regulator of erythroid cell maturation [11], and miR-223 induces myeloid differentiation [12]. Both miR-451 and miR-223 are thought to act as tumor suppressors, and the low plasma levels of these miRNAs in higher-risk MDS patients may contribute to the promotion of leukemic cell growth. In AML, Gentner et al. found significantly higher miR-223 levels in blasts from patients with a favorable prognosis, whereas patients with low miR-223 expression showed worse outcomes [13]. Notably, we previously detected overexpression of cellular miR-451 and miR-223 in CD34+ cells in MDS patients with del(5q) [14], supporting our finding that high expression of these miRNAs is associated with a favorable prognosis in MDS.

There is a great interest in revealing the origin of circulating miRNAs in hematopoietic diseases. For example, Stamatopoulos et al. [15] showed that high levels of serum miR-150 in chronic lymphocytic leukemia (CLL) are linked to lymphocytosis and that cellular miR-150 could be regulated by its release from leukemic cells into the extracellular space. However, they could not definitively demonstrate the correlation between cellular and serum miR-150 levels, suggesting that multiple cells might release miR-150. We also attempted to trace the origin of deregulated miRNAs and tested whether cytopenia (one of the main features of MDS) is linked to the reduction of plasma levels of the miRNAs enriched in the cytopenic lineages (e.g., miR-451a is enriched in erythrocytes [11], miR-223 in myeloid cells [12], miR-27 in granulocytes [16], and miR-199 in platelets [17]). However, we did not observe any correlation between miRNA plasma levels and blood count values. Furthermore, we tested the myeloblastic cell population as another source of circulating miRNAs in MDS. We previously measured miRNA expression profiles in CD34+ MDS cells [10] (the majority of MDS myeloblasts are CD34+ [18]) and here we used these data to assess the correlation between cellular and plasma miRNA levels. However, the levels of plasma miRNAs did not reflect their levels in CD34+ cells. To sum up, these data suggest that miRNA release into MDS plasma is probably not a cell-specific but rather a miRNAs-specific process. In this context, Pigali et al. [19] previously reported that released miRNAs may not reflect the abundance of miRNAs in the cell of origin, suggesting that miRNAs are retained or released selectively and that extracellular miRNAs should be considered independent of cellular miRNA abundance when considering diagnostic markers.

In conclusion, the miRNA profiling performed on MDS plasma provides new information about the phenomenon of circulating miRNAs in MDS pathophysiology. Our data indicate that the specific miRNA signature in MDS plasma may predict patient outcomes independent of the currently used scoring systems. In particular, decreases in the levels of miR451a and miR-223-3p appear to be potential indicators of an adverse prognosis in MDS. Further investigations to examine the plasma levels of these miRNAs in an independent series of patients and to monitor them over the course of disease therapy are needed to evaluate the applicability of these molecules in MDS prognostication.

Supplementary information is available in the online version of the paper.

Acknowledgements: The authors thank Prof Kyra Michalova (Center of Oncocytogenetics, Faculty Hospital and First Faculty of Medicine, Charles University, Prague) for the cytogenetic data. This work was supported by grant 16-33617A from the Ministry of Health of the Czech Republic.

\section{References}

1] YUAN A, FARBER EL, RAPOPORT AL, TEJADA D, DENISKIN $R$ et al. Transfer of microRNAs by embryonic stem cell microvesicles. PLoS One 2009; 4: e4722. https://doi. org/10.1371/journal.pone.0004722

[2] FONSATO V, COLLINO F, HERRERA MB, CAVALLARI C, DEREGIBUS MC et al. Human liver stem cell-derived microvesicles inhibit hepatoma growth in SCID mice by delivering antitumor microRNAs. Stem Cells 2012; 30: 1985-1998. https://doi.org/10.1002/stem.1161

[3] UMEZU T, OHYASHIKI K, KURODA M, OHYASHIKI JH. Leukemia cell to endothelial cell communication via exosomal miRNAs. Oncogene 2013; 32: 2747-2755. https://doi. org/10.1038/onc.2012.295

[4] KIM Y, CHEONG JW, KIM YK, EOM JI, JEUNG HK et al. Serum microRNA-21 as a potential biomarker for response to hypomethylating agents in myelodysplastic syndromes. PLoS One 2014; 9: e86933. https://doi.org/10.1371/journal. pone.0086933

[5] ZUO Z, CALIN GA, DE PAUlA HM, MEDEIROS LJ, FERNANDEZ $\mathrm{MH}$ et al. Circulating microRNAs let-7a and miR-16 predict progression-free survival and overall survival in patients with myelodysplastic syndrome. Blood 2011; 118: 413-415. https://doi.org/10.1182/blood-2011-01-330704

[6] ZUO Z, MAITI S, HU S, LOGHAVI S, CALIN GA et al. Plasma circulating-microRNA profiles are useful for assessing prognosis in patients with cytogenetically normal myelodysplastic syndromes. Mod Pathol 2015; 28: 373-382. https://doi. org/10.1038/modpathol.2014.108

[7] BLONDAL T, JENSBY NIELSEN S, BAKER A, ANDREASEN D, MOURITZEN $P$ et al. Assessing sample and miRNA profile quality in serum and plasma or other biofluids. Methods 2013; 59: S1-S6. https://doi.org/10.1016/j.ymeth.2012.09.015

[8] FILKOVA M, ARADI B, SENOLT L, OSPELT C, VETTORI $S$ et al. Association of circulating miR-223 and miR-16 with disease activity in patients with early rheumatoid arthritis. Ann Rheum Dis 2014; 73: 1898-1904. https://doi.org/10.1136/ annrheumdis-2012-202815

[9] SAEED AI, SHAROV V, WHITE J, LI J, LIANG W et al. TM4: a free, open-source system for microarray data management and analysis. Biotechniques 2003; 34: 374-378.

[10] DOSTALOVA MERKEROVA M, KREJCIKZ, VOTAVOVA H, BELICKOVA M, VASIKOVA A et al. Distinctive microRNA expression profiles in CD34+ bone marrow cells from patients 
with myelodysplastic syndrome. Eur J Hum Genet 2011; 19: 313-319. https://doi.org/10.1038/ejhg.2010.209

[11] BRUCHOVA H, YOON D, AGARWAL AM, MENDELL J, PRCHAL JT. Regulated expression of microRNAs in normal and polycythemia vera erythropoiesis. Exp Hematol 2007; 35: 1657-1667. https://doi.org/10.1016/j.exphem.2007.08.021

[12] ZHUANG G, MENG C, GUO X, CHERUKU PS, SHI L et al. A novel regulator of macrophage activation: miR-223 in obesity-associated adipose tissue inflammation. Circulation 2012; 125: 2892-2903. https://doi.org/10.1161/CIRCULATIONAHA.111.087817

[13] GENTNER B, POCHERT N, ROUHI A, BOCCALATTE F, PLATI T et al. MicroRNA-223 dose levels fine tune proliferation and differentiation in human cord blood progenitors and acute myeloid leukemia. Exp Hematol 2015; 43: 858-868.e7. https://doi.org/10.1016/j.exphem.2015.05.018

[14] VOTAVOVA H, GRMANOVA M, DOSTALOVA MERKEROVA M, BELICKOVA M, VASIKOVA A et al. Differential expression of microRNAs in CD34+ cells of 5q- syndrome. J Hematol Oncol 2011, 4: 1. https://doi.org/10.1186/1756-8722-4-1

[15] STAMATOPOULOS B, VAN DAMME M1, CROMPOT E, DESSARS B, HOUSNI HE et al. Opposite Prognostic
Significance of Cellular and Serum Circulating MicroRNA-150 in Patients with Chronic Lymphocytic Leukemia. Mol Med 2015; 21: 123-133. https://doi.org/10.2119/mol$\underline{\text { med.2014.00214 }}$

[16] FENG J, IWAMA A, SATAKE M, KOHU K. MicroRNA-27 enhances differentiation of myeloblasts into granulocytes by post-transcriptionally downregulating Runx1. Br J Haematol 2009; 145: 412-423. https://doi.org/10.1111/j.13652141.2009.07632.x

[17] PLE H, LANDRY P, BENHAM A, COARFA C, GUNARATNE $\mathrm{PH}$ et al. The repertoire and features of human platelet microRNAs. PLoS One 2012; 7: e50746. https://doi. org/10.1371/journal.pone.0050746

[18] DE SMET D, TRULLEMANS F, JOCHMANS K, RENMANS W, SMET L et al. Diagnostic potential of CD34+ cell antigen expression in myelodysplastic syndromes. Am J Clin Pathol 2012; 138: 732-743. https://doi.org/10.1309/ AJCPAGVO27RPTOTV

[19] PIGATI L, YADDANAPUDI SCS, IYENGAR R, KIM DJ, HEARN SA et al. Selective Release of MicroRNA Species from Normal and Malignant Mammary Epithelial Cells. PLoS One 2010; 5: e13515. https://doi.org/10.1371/journal.pone.0013515 

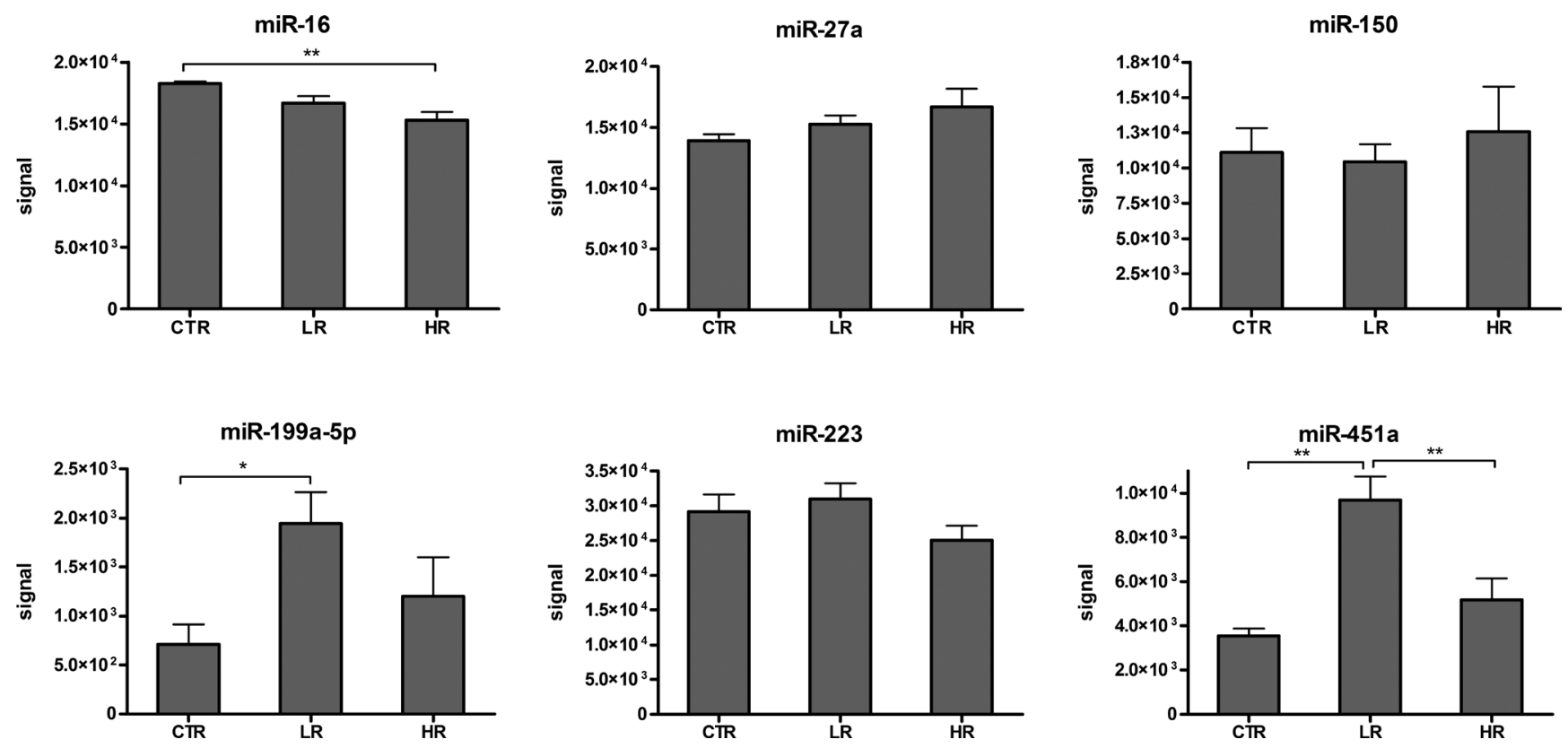

Supplementary Figure 1. MiRNA level in CD34+ MDS cells. Relative expression of miR-16-5p, miR-27a-3p, miR-150-5p, miR-199a-5p, miR-223-3p, and miR-451a in CD34+ MDS cells were calculated based on microarray data [10]. CTR - controls, LR - lower-risk, HR - higher-risk MDS patients, * $P<.05,{ }^{* *} P<.01,{ }^{* * *} P<.001$. 
SI Table 1. MiRNAs significantly deregulated $(P<.01)$ in MDS plasma compared to controls.

\begin{tabular}{|c|c|c|c|c|}
\hline \multirow[t]{2}{*}{ miRNA } & \multicolumn{2}{|c|}{ Signal intensity (mean) } & \multirow[t]{2}{*}{ Fold change } & \multirow[t]{2}{*}{$P$ value } \\
\hline & CTR & MDS & & \\
\hline miR-4655-3p & 4.2 & 39.1 & 9.41 & .00004 \\
\hline miR-3648 & 4.2 & 58.4 & 14.05 & .00001 \\
\hline miR-5585-3p & 4.2 & 53.7 & 12.92 & .00002 \\
\hline miR-4497 & 6.9 & 92.9 & 13.39 & .0003 \\
\hline miR-575 & 98.0 & 646.4 & 6.59 & .0022 \\
\hline $\operatorname{miR}-548 q$ & 8.9 & 96.6 & 10.86 & .0004 \\
\hline miR-3194-5p & 4.2 & 40.0 & 9.63 & .00004 \\
\hline miR-4476 & 6.5 & 40.3 & 6.23 & .0019 \\
\hline miR-6068 & 393.5 & 1774.3 & 4.51 & .0048 \\
\hline miR-1227-5p & 137.3 & 750.8 & 5.47 & .0039 \\
\hline miR-572 & 92.2 & 519.7 & 5.64 & .0033 \\
\hline miR-5001-5p & 192.5 & 669.7 & 3.48 & .0084 \\
\hline miR-371b-5p & 363.4 & 1532.5 & 4.22 & .0061 \\
\hline $\operatorname{miR}-4787-5 p$ & 207.2 & 814.6 & 3.93 & .0057 \\
\hline $\operatorname{miR}-4745-5 p$ & 15.7 & 59.7 & 3.80 & .0061 \\
\hline miR-188-5p & 9.7 & 35.7 & 3.68 & .0068 \\
\hline miR-4788 & 18.3 & 71.1 & 3.89 & .0060 \\
\hline miR-4632-5p & 5.3 & 22.4 & 4.20 & .0013 \\
\hline miR-6723-5p & 4.2 & 24.0 & 5.78 & .0003 \\
\hline $\operatorname{miR}-4270$ & 120.7 & 306.3 & 2.54 & .0092 \\
\hline miR-1202 & 238.9 & 660.7 & 2.77 & .0087 \\
\hline miR-6085 & 83.2 & 228.3 & 2.74 & .0099 \\
\hline miR-150-5p & 17.0 & 90.3 & 5.30 & .0005 \\
\hline miR-6125 & 3605.0 & 9097.4 & 2.52 & .0094 \\
\hline miR-198 & 4.9 & 12.4 & 2.54 & .0064 \\
\hline miR-1273f & 4.2 & 15.6 & 3.76 & .0023 \\
\hline $\mathrm{miR}-4516$ & 2510.0 & 4894.8 & 1.95 & .0083 \\
\hline miR-483-5p & 9.2 & 26.9 & 2.92 & .0047 \\
\hline miR-3138 & 8.6 & 24.9 & 2.88 & .0041 \\
\hline miR-4454 & 23778.6 & 11048.1 & 0.46 & .0081 \\
\hline miR-197-3p & 126.8 & 70.7 & 0.56 & .0045 \\
\hline $\operatorname{miR}-423-5 p$ & 150.6 & 56.8 & 0.38 & .0040 \\
\hline miR-484 & 36.8 & 11.2 & 0.31 & .0016 \\
\hline miR-92a-3p & 1092.1 & 410.1 & 0.38 & .0018 \\
\hline miR-106b-5p & 157.3 & 70.4 & 0.45 & .0062 \\
\hline miR-3135b & 352.4 & 120.3 & 0.34 & .0006 \\
\hline miR-30d-5p & 469.8 & 57.1 & 0.12 & .0018 \\
\hline miR-342-3p & 58.9 & 11.2 & 0.19 & .0020 \\
\hline $\operatorname{miR}-451 \mathrm{a}$ & 6068.9 & 1351.3 & 0.22 & .0065 \\
\hline miR-101-3p & 34.9 & 10.6 & 0.30 & .0059 \\
\hline miR-140-3p & 37.7 & 9.3 & 0.25 & .0024 \\
\hline miR-199a-3p & 155.2 & 49.1 & 0.32 & .0094 \\
\hline miR-142-5p & 96.7 & 28.6 & 0.30 & .0064 \\
\hline
\end{tabular}




\begin{tabular}{|l|r|r|r|r|}
\hline miR-142-3p & 466.4 & 112.2 & 0.24 & .0086 \\
\hline miR-320b & 399.7 & 45.2 & 0.11 & .0005 \\
\hline miR-320e & 520.3 & 55.1 & 0.11 & .0005 \\
\hline miR-320d & 768.8 & 67.8 & 0.09 & .0006 \\
\hline miR-320a & 153.9 & 23.8 & 0.15 & .0014 \\
\hline miR-4655-3p & 4.2 & 39.1 & 9.41 & .00004 \\
\hline miR-3648 & 4.2 & 58.4 & 14.05 & .00001 \\
\hline miR-5585-3p & 4.2 & 53.7 & 12.92 & .00002 \\
\hline
\end{tabular}

SI Table 2. MiRNAs significantly altered $(P<.01)$ between lower-risk and higher-risk MDS.

\begin{tabular}{|c|c|c|c|c|}
\hline miRNA & \multicolumn{2}{|c|}{ Signal intensity (mean) } & Fold change & $P$ value \\
\hline & Lower-risk MDS & Higher-risk MDS & & \\
\hline miR-548q & 49.1 & 160.1 & 3.26 & .0069 \\
\hline $\operatorname{miR}-4739$ & 70.8 & 193.3 & 2.73 & .0026 \\
\hline miR-4721 & 21.7 & 90.3 & 4.16 & .0005 \\
\hline miR-188-5p & 21.6 & 54.6 & 2.53 & .0062 \\
\hline miR-4499 & 18.1 & 36.2 & 2.00 & .0028 \\
\hline miR-623 & 7.7 & 34.4 & 4.44 & .0095 \\
\hline miR-20b-5p & 45.1 & 17.9 & 0.40 & .0064 \\
\hline $\operatorname{miR}-27 a-3 p$ & 30.4 & 4.7 & 0.15 & .0084 \\
\hline miR-20a-5p & 180.6 & 85.3 & 0.47 & .0060 \\
\hline miR-17-5p & 102.7 & 41.8 & 0.41 & .0081 \\
\hline miR-30e-5p & 36.0 & 11.8 & 0.33 & .0020 \\
\hline miR-16-5p & 744.0 & 255.9 & 0.34 & .0018 \\
\hline miR-4306 & 127.0 & 49.6 & 0.39 & .0030 \\
\hline miR-185-5p & 96.6 & 31.8 & 0.33 & .0014 \\
\hline miR-93-5p & 59.1 & 26.5 & 0.45 & .0025 \\
\hline miR-223-3p & 1521.8 & 339.8 & 0.22 & .0091 \\
\hline miR-27b-3p & 311.9 & 74.0 & 0.24 & .0097 \\
\hline miR-548q & 49.1 & 160.1 & 3.26 & .0069 \\
\hline miR-4739 & 70.8 & 193.3 & 2.73 & .0026 \\
\hline
\end{tabular}

